\title{
Brain-derived neurotrophic factor levels in newly diagnosed patients with bipolar disorder, their unaffected first-degree relatives and healthy controls
}

Nanna Aagaard Petersen, Marc Østergaard Nielsen, Klara Coello, Sharleny Stanislaus, Sigurd Melbye, Hanne Lie Kjærstad, Kimie Stefanie Ormstrup Sletved, Roger S. McIntyre, Ruth Frikke-Smith, Maj Vinberg and Lars Vedel Kessing

\section{Background}

Brain-derived neurotrophic factor (BDNF), which facilitates neuroplasticity and synaptogenesis, may be decreased in bipolar disorder, but has not been systematically investigated in people with newly diagnosed bipolar disorder and unaffected firstdegree relatives.

\section{Aims}

To compare BDNF levels in patients with newly diagnosed bipolar disorder, their unaffected first-degree relatives and healthy controls.

\section{Method}

The study investigated plasma BDNF levels in patients $(n=371)$ with newly diagnosed bipolar disorder, their unaffected firstdegree relatives $(n=98)$ and healthy controls $(n=200)$ using enzyme-linked immunosorbent assay. We further investigated associations between BDNF levels and illness-related variables and medication status.

\section{Results}

BDNF levels were found to be $22.0 \%(95 \% \mathrm{Cl} 1.107-1.343)$ higher in patients with bipolar disorder compared with healthy controls $(P<0.001)$ and $15.6 \%$ higher in unaffected first-degree relatives compared with healthy controls $(95 \% \mathrm{Cl} 1.007-1.327, P=0.04)$, when adjusting for age and gender. Further, BDNF levels were positively associated with duration of illness at a trend level $(P=0.05)$, age $(P=0.001)$ and use of anti-epileptic medication $(P=0.05)$

\section{Conclusions}

These findings suggest that BDNF levels are not decreased in the early stages of bipolar disorder and in unaffected first-degree relatives contrasting with prior findings during later stages of the illness.

\section{Keywords}

Bipolar disorder; brain-derived neurotrophic factor; recent onset; unaffected relatives.

\section{Copyright and usage}

(c) The Author(s), 2021. Published by Cambridge University Press on behalf of the Royal College of Psychiatrists. This is an Open Access article, distributed under the terms of the creative commons Attribution licence (http://creativecommons.org/ licenses/by/4.0/), which permits unrestricted re-use, distribution, and reproduction in any medium, provided the original work is properly cited.

\section{Background}

Bipolar disorder is often a progressive disorder with a high risk of recurrence of depressive and manic episodes ${ }^{1}$ and with functional disability, decreased quality of life and a reduced life expectancy of 8-12 years. ${ }^{2}$ It has been hypothesised that the potential pathology of bipolar disorder may be because of impairments in neuroplasticity. ${ }^{3}$ Brain-derived neurotrophic factor (BDNF) is a member of the neurotropic family and is widely distributed in the central nervous system. BDNF plays a central role in the survival and function of neurons in the brain ${ }^{4}$ and furthermore, influences the neuronal plasticity of the brain as involved in cellular models of memory and learning. Lower BDNF levels could therefore result in reduced neuronal activity, loss of normal plasticity and damage to neurons in the brain. ${ }^{5}$ BDNF has been demonstrated to cross the blood-brain barrier $^{6}$ and peripheral blood levels are found to be decreased in major depression ${ }^{7}$ and schizophrenia. ${ }^{8}$

\section{Use of BDNF levels as a potential biomarker}

As a result of these neuroplastic qualities BDNF levels have been explored in patients with bipolar disorder, and based on reviews and meta-analyses BDNF is suggested as a potential biomarker related to neuroprogression and disease activity. ${ }^{9,10}$ Two recent meta-analyses including 53 studies $^{11}$ and 52 studies, ${ }^{10}$ found that patients with bipolar disorder tend to have lower peripheral BDNF levels during both depressive and manic episodes compared with healthy controls, and that BDNF levels may return to normal in euthymic states.

In conclusion, changes in BDNF levels seem to be involved in the pathogenesis of bipolar disorder and could reflect trait markers for the disorder, being present prior to and following onset of the illness and potentially decrease further with clinical progression of the illness. ${ }^{12}$ However, no large-scale study has investigated BDNF levels in people with newly diagnosed bipolar disorder and their unaffected first-degree relatives.

\section{Aims}

The aim of our present study was to investigate plasma BDNF levels in patients newly diagnosed with bipolar disorder, and their unaffected first-degree relatives, in comparison with healthy controls. We hypothesised that patients newly diagnosed with bipolar disorder would have lower BDNF levels compared with healthy controls without a family history of bipolar disorder. Furthermore, we hypothesised that first-degree relatives of patients with bipolar disorder would express intermediary BDNF levels. 


\section{Method}

\section{Study design}

The present cross-sectional study analyses baseline data from the ongoing, longitudinal Bipolar Illness Onset (BIO) study, which aims to identify composite biomarkers for bipolar disorder. A full research protocol has been published for the BIO cohort study. ${ }^{13}$ Recruitment into the BIO study started in June 2015 and ended in November 2019. The study was approved by the Committee on Health Research Ethics of the Capital Region of Denmark (protocol No. H-7-2014-007) and the Danish Data Protection Agency, Capital Region of Copenhagen (RHP-2015-023). Written informed consent was provided by all participants. The study complied with the Declaration of Helsinki principles.

\section{Participants}

\section{Patients with bipolar disorder}

Patients were recruited from the Copenhagen Affective Disorder Clinic, which covers the entire greater Copenhagen catchment area (Region Hovedstaden). The Copenhagen Affective Disorder Clinic covers a catchment area of 1.6 million people, including all psychiatric centres in the region, and provides assessment and treatment for patients with newly diagnosed/first-episode bipolar disorder (i.e. for patients where the diagnosis of a single manic episode/bipolar disorder is made for the first time). All patients referred to the Copenhagen Affective Disorder Clinic with newly diagnosed bipolar disorder/a diagnosis of a single manic episode were invited to participate in the BIO study. Inclusion criteria were an ICD-10 diagnosis of bipolar disorder or a single manic episode and age 15-70 years. Patients were excluded if they had bipolar disorder secondary to brain injury.

\section{Unaffected first-degree relatives}

After consent was obtained from the identified patients with bipolar disorder, their siblings and offspring (age $>15$ years) were invited to participate in the BIO study. Inclusion criteria included being a firstdegree relative of an included patient with bipolar disorder between the ages of 15-70 years. Siblings diagnosed with bipolar disorder or schizophrenia, current substance misuse, half-siblings and adopted off-springs/siblings were not included.

\section{Healthy controls}

Healthy controls were recruited among blood donors from the Blood Bank at Rigshospitalet Copenhagen, Denmark. The donors were contacted in the waiting room on random days. The inclusion criterion was being between the ages of 15 and 70 years, and exclusion criteria included having a personal history or first-degree family history of psychiatric disorder that required psychiatric treatment.

\section{Diagnostic and clinical assessment}

Diagnostic assessment and an assessment of the patient's current affective state was performed by specialists in psychiatry at the Copenhagen Affective Disorder Clinic according to the ICD-10 and DSM-IV-criteria ${ }^{14}$ for bipolar disorder type I and bipolar disorder type II. After informed consent, $\mathrm{PhD}$ students in medicine or psychology subsequently confirmed the bipolar disorder diagnosis using the Schedules for Clinical Assessment in Neuropsychiatry (SCAN). ${ }^{15}$ Moreover, severity of depressive and manic symptoms was assessed according to the 17-item Hamilton Rating Scale for Depression Scale-17 items (HRSD-17) ${ }^{16}$ and the Young Mania Rating Scale (YMRS), respectively. ${ }^{17}$ Medication status, sleep patterns, alcohol intake, smoking habits and exercise patterns were recorded.

\section{Blood sample collection and analysis}

Fasting blood samples were collected at the Department of Clinical Biochemistry, Rigshospitalet in a resting state between $07.30 \mathrm{~h}$ and $10.00 \mathrm{~h}$ on the same day as the clinical assessment. A total of $5 \mathrm{~mL}$ of blood was drawn by venepuncture into EDTA (ethylenediaminetetraacetic acid)-containing vacuum tube $\left(\right.$ Vacuette $\left.^{\circ}\right)$. Afterwards, EDTA was centrifuged for $30 \mathrm{~min}$ at $1590 \mathrm{~g}$ and $4^{\circ} \mathrm{C}$ for $15 \mathrm{~min}$. Plasma was aliquoted into Eppendorf ${ }^{\circ}$ tubes and kept frozen at $-80^{\circ} \mathrm{C}$ until plasma BDNF levels were assayed. Plasma concentration of BDNF was measured in duplicate using a sandwich enzymelinked immunosorbent assay (ELISA) kit (R\&D Systems, USA, Cat. No DBNT00). The lower limit of detection for BDNF was $15.6 \mathrm{pg} /$ $\mathrm{mL}$. Intra-assay coefficient of variance $(\mathrm{CV})$ was $4.0 \%$ and the interassay CV was $16.4 \%$.

Seven blood samples (for five patients with bipolar disorder and two unaffected relatives) were either below the limit of detection or had too little material to be analysed and therefore could not be included. The laboratory staff was masked to the participant's diagnostic status, and the samples were randomly assigned across assays.

\section{Statistical analyses}

To test differences between patients with bipolar disorder, their unaffected first-degree relatives and healthy controls, SPSS version 25 was used (SPSS for Windows). The level of significance was set at $P<0.05$. Categorical clinical demographic variables were analysed with the Chi-square test, and the remaining variables by independent sample $t$-test. When assumption of normality was not met, continuous data were analysed using the non-parametric KruskalWallis test and presented as median and quartiles.

Linear mixed-effect models were used in the main analyses and were performed as two separate sets (model A and model B). The analysis strategy was planned a priori. First, one set (model A) was performed as a between-group comparison (patients with bipolar disorder, unaffected relatives and healthy controls) in an unadjusted linear mixed-effect model, with BDNF levels as the dependent variable, and family relationship as the random effect, to account for the correlation between the related participants. Second, gender and age were added as covariates (model A-1), and third HRSD-17 (total score), YMRS (total score), alcohol consumption (>14 units per week/ $\leq 14$ units per week) and smoking status (yes/no) in a fully adjusted model (model A-2), as smoking status and alcohol consumption has been suggested to influence BDNF levels when categorised as described above. ${ }^{18}$

In a second set (model B), we conducted multiple linear regression analyses within patients. The first adjusted model (model B-1) included age and gender and severity according to HRSD-17 and YMRS (total scores) as independent variables and BDNF levels as the dependent variable. In a second model, illness duration (years) and current psychiatric medication treatment (yes/no) with antidepressant, anti-epileptic, antipsychotic and lithium as covariates were included, in addition to affective state, as independent variables (model B-2). Afterward, the analyses were repeated but replacing the four treatment variables with the categorical variable 'receiving psychotropic medication' versus 'medication-free'.

Finally, a number of post hoc analyses were conducted: bivariate explorative analyses to further explore the association between BDNF levels and sociodemographic and clinical variables, using correlational analyses as well as factor analysis, and $K$-means and Hierarchical cluster analysis. 
For all parametric tests, BDNF levels were transformed by logarithm base to 10 , and presented as back-transformed values, estimate $B$, representing the mean ratio between variables.

\section{Results}

\section{Demographic and clinical characteristics}

A total of 371 patients with bipolar disorder, 98 unaffected firstdegree relatives and 200 healthy controls were included in the study. The majority of patients with bipolar disorder (58.6\%) were in full remission defined as a score $<8$ on the HRSD-17 and the YMRS rating scales on the day of inclusion. Clinical and demographic variables of the participants are shown in Table 1.

The included 98 unaffected first-degree relatives were relatives of 83 patients with bipolar disorder, as 15 patients had two firstdegree relatives included. As can be seen in Table 1, there was no statistically significant difference between patients with bipolar disorder, unaffected first-degree relatives and healthy control individuals regarding gender, height, weight and body mass index. There were minor, although statistically significant, differences in age between patients with bipolar disorder and healthy controls (29.1 v. 27.4 years, $P=0.008$ ) and between unaffected first-degree relatives and healthy controls (26.8 v. 27.4 years, $P=0.03)$. Patients with bipolar disorder and their unaffected first-degree relatives had slightly fewer years of education and compared with healthy controls. Furthermore, patients with bipolar disorder and their unaffected first-degree relatives had significantly lower alcohol consumption compared with healthy controls $(P=0.008$ and $P=0.027$, respectively). The number of current smokers was significantly higher among patients with bipolar disorder compared with unaffected first-degree relatives $(P<0.001)$ and healthy controls $(P<0.001)$.

\section{Plasma BDNF levels in patients with bipolar disorder, their unaffected first-degree relatives and healthy controls}

As illustrated in Fig. 1, in the unadjusted linear mixed-effect model, we found that patients with bipolar disorder had a $22.0 \%$ higher BDNF level compared with healthy controls $(B=1.220,95 \%$ CI $1.104-1.343, P<0.001)$, whereas the $8.1 \%$ higher level as compared with unaffected first-degree relatives was not statistically significant ( $B=1.081,95 \%$ CI $0.955-1.227, P=0.214)$. Moreover, unaffected first-degree relatives had a $12.7 \%$ higher BDNF level than healthy controls, but this failed to reach significance $(B=1.127,95 \% \mathrm{CI}$ 0.979-1.294, $P=0.094$ ).

As can be seen in Table 2, in the model 1 adjusted for group, age and gender, the BDNF levels remained statistically significantly higher in patients with bipolar disorder compared with healthy controls $(B=1.220,95 \%$ CI $1.107-1.343, P<0.001)$. Additionally, BDNF levels were $15.6 \%$ higher in unaffected first-degree relatives compared with healthy controls $(B=1.156,95 \%$ CI $1.007-1.327$, $P=0.041)$. There was no difference between patients with bipolar disorder and unaffected first-degree relatives $(B=1.054,95 \% \mathrm{CI}$ $0.931-1.198, P=0.402)$. In this adjusted model, increasing age was associated with statistically significantly higher levels of $\operatorname{BDNF}(B=1.008,95 \%$ CI 1.003-1.013, $P=0.001)$. Exclusion of participants with BDNF levels higher than two s.d. above the mean (22 patients, 2 unaffected first-degree relatives and 2 healthy controls) did not change the results.

In the fully adjusted model 2 including group, age, gender, HRSD-17 (total score), YMRS (total score), alcohol consumption and smoking status, patients with bipolar disorder had a statistically significant higher BDNF level of $22.5 \%$ compared with healthy controls $(B=1.225,95 \%$ CI 1.067-1.406, $P=0.004$, Table 2). Unaffected first-degree relatives had a statistically significant $15.3 \%$ higher BDNF level compared with healthy controls $(B=$ 1.153 , 95\% CI $0.998-1.334, P=0.05)$. No difference between patients with bipolar disorder and unaffected first-degree relatives were found ( $B=1.026,95 \%$ CI $0.912-1.233, P=0.45)$. In the fully adjusted model, age was positively associated with BDNF levels ( $B=1.008,95 \%$ CI 1.003-1.0131, $P=0.002)$, but there was no association with the other covariates. These results did not change when excluding all participants with HRSD-17 $\geq 14$ and YMRS $\geq 14$. When participants with BDNF levels higher than two s.d.s above the mean were excluded, the significant difference between patients with bipolar disorder and healthy controls, and unaffected firstdegree relatives and healthy controls, lost significance below the 0.05 level $(P=0.07$ and $P=0.07$, respectively).

\section{Associations between affective state, illness duration, medication and BDNF levels in newly diagnosed patients with bipolar disorder}

As can be seen from Table 3, multiple linear regression analysis revealed no statistically significant association between BDNF levels and age, gender and symptom severity according to HRSD17 and YMRS (total scores). However, multiple linear regression analysis with BDNF levels as the dependent variable and illness duration (years), current psychiatric medication treatment (yes/no) with antidepressants, anti-epileptics, antipsychotics and lithium as the independent variables, revealed a statistically significant association between BDNF levels and anti-epileptic medication (unstandardised $B=1.135,95 \%$ CI $1.000-1.288, P=0.048$ ). Excluding patients with BDNF levels two s.d.s above the mean did not change the results. However, only $4.7 \%$ variance was explained.

Replacing the four treatment variables with the categorical variable 'medication-free' versus 'receiving psychotropic medication' revealed statistically significant lower BDNF levels in patients not receiving any medication $(P=0.04)$.

\section{Post hoc explorative analyses}

To exclude methodological biases, exploration of variance in plasma storage time did not reveal significance (Spearman correlation, $P=$ 0.1 ). However, there was a statistically significant positive association between longer illness duration (years) and higher BDNF levels in participants with bipolar disorder $(R=1.000, P=0.05)$. Overall, independent sample $t$-tests revealed no significant difference in BDNF levels, neither between male and female gender $(P=0.508)$ nor in analysis of participant groups (bipolar disorder $P=0.280$, unaffected relatives $P=0.152$, healthy controls $P=0.08$ ). Furthermore, when only including patients who did not receive any psychotropic medication $(n=64)$ in the mixed linear models, the difference in BDNF levels between patients with bipolar disorder and healthy controls lost significance $(P=0.4)$.

\section{Discussion}

The BIO study is the first study to investigate BDNF in patients who are newly diagnosed with bipolar disorder or with first-episode bipolar disorder and their unaffected first-degree relatives in comparison with healthy controls. The findings did not support our first hypothesis, that newly diagnosed patients with bipolar disorder would have lower BDNF levels compared with healthy controls. In contrast, we found significantly higher BDNF levels in patients with bipolar disorder compared with healthy controls that could not be explained by alcohol consumption, smoking status or subsyndromal symptoms. Further, BDNF levels increased with illness duration. 


\begin{tabular}{|c|c|c|c|c|c|c|}
\hline & $\begin{array}{l}\text { Bipolar disorder } \\
\text { group }(n=371)\end{array}$ & $\begin{array}{l}\text { Unaffected relative } \\
\text { group }(n=98)\end{array}$ & $\begin{array}{l}\text { Healthy control } \\
\text { group }(n=200)\end{array}$ & $\begin{array}{l}\text { Bipolar disorder } \\
\text { group versus } \\
\text { unaffected relative } \\
\text { group, } P\end{array}$ & $\begin{array}{l}\text { Bipolar disorder } \\
\text { group versus } \\
\text { healthy control } \\
\text { group, } P\end{array}$ & $\begin{array}{l}\text { Unaffected relative } \\
\text { group versus } \\
\text { healthy control } \\
\text { group, } P\end{array}$ \\
\hline Age, years: median (IQR) & $29.1(24.2-36.8)$ & $26.8(22.8-31.8)$ & $27.4(24.3-36.1)$ & 0.008 & 0.387 & 0.03 \\
\hline Gender, female: $n$ (\%) & $241(65.0)$ & $56(57.1)$ & $128(64.0)$ & 0.153 & 0.819 & 0.253 \\
\hline Education, total years: mean (IQR) & $15(12.5-17)$ & $15(13-17)$ & $16(14.6-17)$ & 0.216 & $<0.001$ & 0.02 \\
\hline HRSD-17, total score: median (IQR) & $9(5-15)$ & $2(0.75-4)$ & $0(0-2)$ & $<0.001$ & $<0.001$ & $<0.001$ \\
\hline YMRS, total score: median (IQR) & $3(0-7)$ & $0(0-2)$ & $0(0-1)$ & $<0.001$ & $<0.001$ & 0.280 \\
\hline Weight, kg: mean (s.d.) IQR & 75.9 (15.6) 43.8 to 124.6 & $74.4(14.2) 51.6$ to 117.2 & 74.3 (13.9) 49.3 to 131.4 & 0.387 & 0.246 & 0.991 \\
\hline Hight, cm: mean (s.d.) IQR & $173.2(9.0) 153$ to 199 & $173.6(9.3) 153$ to 196 & $174.3(8.8) 157$ to 200 & 0.724 & 0.169 & 0.517 \\
\hline Body mass index, mean (s.d.) IQR & $25.2(4.6) 15$ to 48 & 24.7 (4.6) 18 to 44 & 24.4 (3.4) 16 to 37 & 0.336 & 0.021 & 0.450 \\
\hline Current smokers, $n$ (\%) & $160(43.8)^{\mathrm{a}}$ & $21(21.9)$ & $21(10.5)$ & $<0.001$ & $<0.001$ & 0.009 \\
\hline Weekly alcohol consumption, units: mean (s.d.) IQR & $4.7(6.8) 0$ to $42^{\mathrm{a}}$ & $4.7(5.6) 0$ to $22^{\mathrm{a}}$ & 6.2 (5.5) 0 to 36 & 0.972 & 0.008 & 0.027 \\
\hline Age at onset, years: mean (s.d.) IQR & $22.6(8.6) 4$ to 60 & - & - & - & - & - \\
\hline Illness duration, years: ${ }^{a}$ mean (s.d.) IQR & 11.6 (8.5) 0 to 45 & - & - & - & - & - \\
\hline Untreated bipolar disorder, years: ${ }^{\mathrm{b}}$ mean (s.d.) IQR & $7.1(8.0)-1$ to 39 & - & - & - & - & - \\
\hline Bipolar disorder type I/type II, $n$ (\%) & $118 / 253(31.8 / 68.2)$ & - & - & - & - & - \\
\hline \multicolumn{7}{|l|}{ Current affective state, $n(\%)$} \\
\hline Full remission & $215(58.6)$ & - & - & - & - & - \\
\hline Mild/moderate depressive episode & $90(24.5)$ & - & - & - & - & - \\
\hline Severe depressive episode & $9(2.4)$ & - & - & - & - & - \\
\hline Manic episode & $1(0.3)$ & - & - & - & - & - \\
\hline Hypomanic episode & $31(8.4)$ & - & - & - & - & - \\
\hline Mixed episode & $19(5.2)$ & - & - & - & - & - \\
\hline Not available & $2(0.5)$ & - & - & - & - & - \\
\hline \multicolumn{7}{|l|}{ Current psychotropic medication ${ }^{c}(n, \%)$} \\
\hline No psychotropic medication & $64(17.3)$ & - & - & - & - & - \\
\hline Antidepressant treatment & $47(12.6)$ & - & - & - & - & - \\
\hline Antipsychotic treatment & $134(36.0)$ & - & - & - & - & - \\
\hline Antiepileptic treatment $(n, \%)$ & $194(52.2)$ & - & - & - & - & - \\
\hline Lithium treatment & $112(30.1)$ & - & - & - & - & - \\
\hline P-BDNF levels, pg/mL: mean (IQR) & 13035.25 (8830.49 to 18723.59 ) & 12385.28 (8519.54 to 17365.56$)$ & 11652.75 (7277.94 to 16605.70$)$ & 0.302 & 0.001 & 00.176 \\
\hline $\begin{array}{l}\text { IQR, interquartile range; HRSD-17, 17-item Hamilton Ratings } \mathrm{SC} \\
\text { a. IIness duration defined as time from first mood episode to i } \\
\text { b. Untreated bipolar disorder defined as time from first, hypom } \\
\text { c. The percentage of current psychotropic medication exceeds }\end{array}$ & $\begin{array}{l}\text { le for Depression; YMRS, Young Mania Ra } \\
\text { clusion date. } \\
\text { anic, manic or mixed episode to time of b } \\
100 \% \text { as several patients with bipolar dis }\end{array}$ & $\begin{array}{l}\text { Scale; P-BNPF, plasma-BNPF. } \\
\text { ar disorder diagnosis. } \\
\text { er received more than one psychopha }\end{array}$ & 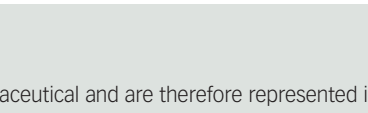 & 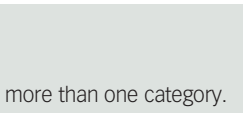 & & \\
\hline
\end{tabular}




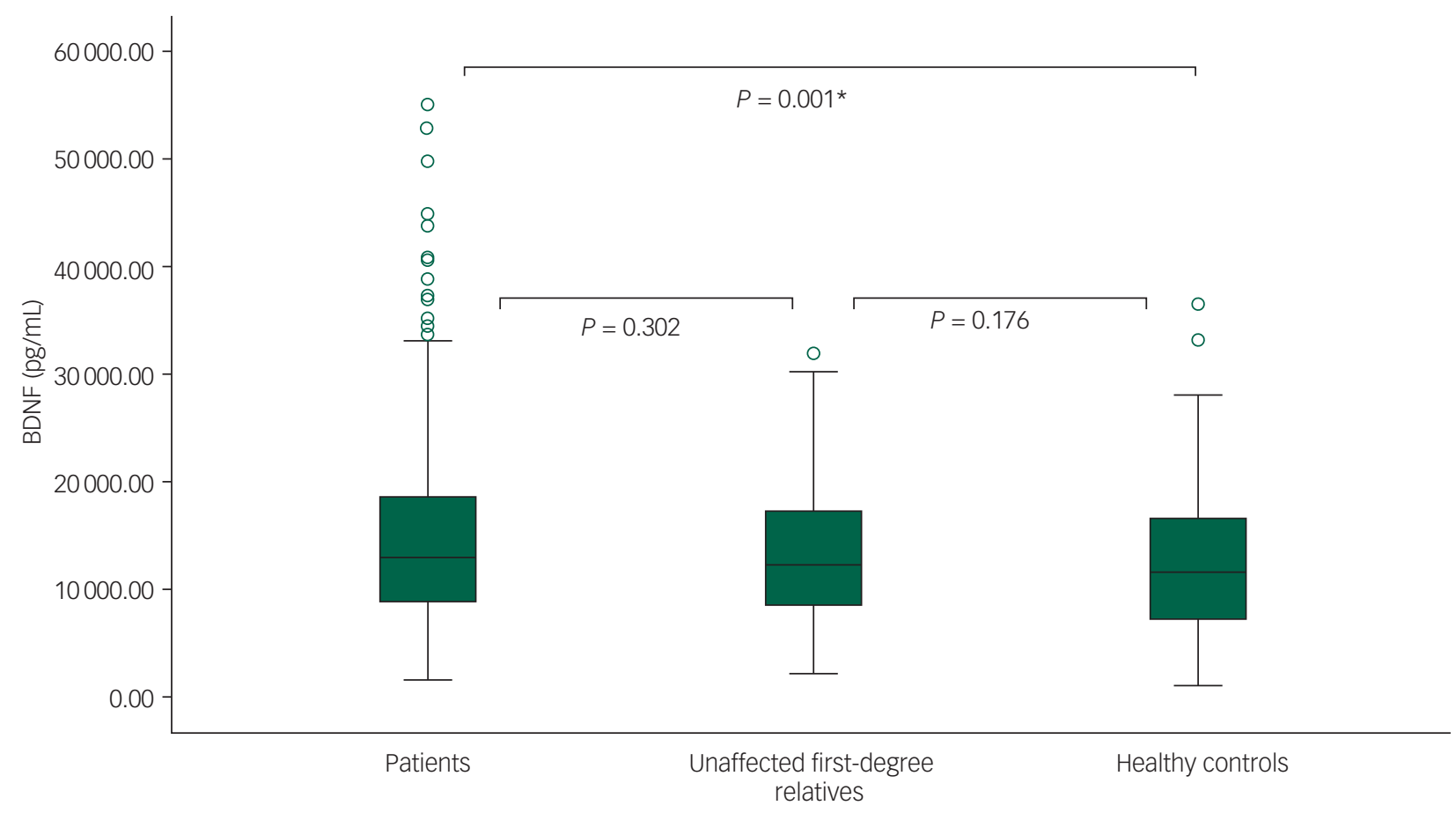

Fig. 1 Box plots comparing brain-derived neurotrophic factor (BDNF) levels $(\mathrm{pg} / \mathrm{mL})$ in newly diagnosed patients with bipolar disorder, their unaffected first-degree-relatives and healthy controls.

The lower and upper hinges represent the first and third quartiles. The upper and lower whiskers extend from the hinge to the largest and lower value, respectively. Data beyond the end of the whiskers are plotted individually. * Statistically significant at 0.05 level.

Similarly, our second hypothesis was also not confirmed as we found significantly higher BDNF levels in unaffected first-degree relatives compared with healthy controls, including when adjusting for age and gender. Overall, increasing age was associated with higher BDNF levels. Furthermore, within patients with bipolar disorder we found a significant association between receiving anti-epileptic medication and higher BDNF levels.

\section{Interpretation of our findings}

In contrast with most prior studies investigating BDNF in patients with more progressed bipolar disorder, ${ }^{10,11}$ we found newly diagnosed patients to have elevated BDNF levels as compared with healthy controls. However, our finding that BDNF levels were positively associated with duration of illness (at a trend level) are in line with the previous meta-analysis from our group that included 35 studies comprising a total of 3798 participants ${ }^{9}$ that found higher levels of BDNF were associated with longer duration of bipolar disorder illness.

It is highly unlikely that the present findings are a result of errors in the blood sample collection or analysis process as these were undertaken according to state of the art. Fasting blood samples were collected in a resting state between $07.30 \mathrm{~h}$ and $10.00 \mathrm{~h}$ and measures of plasma concentration of BDNF were made in duplicate

\begin{tabular}{|c|c|c|c|}
\hline & $B$ & $95 \% \mathrm{Cl}$ & $P$ \\
\hline \multicolumn{4}{|l|}{ Model $1^{\text {a }}$} \\
\hline Bipolar disorder versus unaffected first-degree relatives & 1.054 & $0.931-1.198$ & 0.402 \\
\hline Bipolar disorder versus healthy controls & 1.220 & $1.107-1.343$ & $<0.001$ \\
\hline Unaffected first-degree relatives versus healthy controls & 1.156 & $1.007-1.327$ & 0.04 \\
\hline Age & 1.008 & $1.003-1.013$ & 0.001 \\
\hline Male versus female gender & 0.998 & $0.911-1.094$ & 0.967 \\
\hline \multicolumn{4}{|l|}{ Model $2^{b}$} \\
\hline Bipolar disorder versus unaffected first-degree relatives & 1.026 & $0.912-1.233$ & 0.445 \\
\hline Bipolar disorder versus healthy controls & 1.225 & $1.067-1.406$ & 0.004 \\
\hline Unaffected first-degree relatives versus healthy controls & 1.153 & $0.998-1.334$ & 0.052 \\
\hline Age & 1.008 & $1.003-1.013$ & 0.002 \\
\hline Male versus female & 0.997 & $0.906-1.098$ & 0.951 \\
\hline Smoking versus not smoking & 0.937 & $0.845-1.038$ & 0.213 \\
\hline Alcohol computation ( $\leq 14$ units per week versus $>14$ units per week) & 0.927 & $0.790-1.088$ & 0.354 \\
\hline 17-item Hamilton Ratings Scale for Depression & 1.003 & $0.995-1.012$ & 0.432 \\
\hline Young Mania Rating Scale & 0.998 & $0.986-1.009$ & 0.683 \\
\hline
\end{tabular}




\begin{tabular}{|c|c|c|c|}
\hline & B & $95 \% \mathrm{Cl}$ & $P$ \\
\hline \multicolumn{4}{|l|}{ Model 1, unstandardised ${ }^{a}$} \\
\hline Age & 1.007 & $1.000-1.012$ & 0.074 \\
\hline Male versus female & 1.091 & $0.964-1.239$ & 0.168 \\
\hline 17-item Hamilton Depression Rating Scale & 1.002 & $0.984-1.009$ & 0.771 \\
\hline Young Mania Rating Scale & 0.995 & $0.984-1.007$ & 0.455 \\
\hline \multicolumn{4}{|l|}{ Model $2^{\mathrm{b}}$} \\
\hline Age & 1.002 & $0.993-1.009$ & 0.794 \\
\hline Male versus female & 1.074 & $0.948-1.219$ & 0.257 \\
\hline Current anti-epileptic medication & 1.135 & $1.000-1.288$ & 0.05 \\
\hline Current antipsychotic medication & 1.040 & $0.920-1.178$ & 0.524 \\
\hline Current antidepressive medication & 1.146 & $0.962-1.361$ & 0.127 \\
\hline Current lithium medication & 1.059 & $0.920-1.219$ & 0.411 \\
\hline Illness duration (years) & 1.005 & $0.995-1.014$ & 0.239 \\
\hline Medication versus medication-free & 0.897 & $0.773-1.040$ & 0.04 \\
\hline
\end{tabular}

using a sandwich ELISA kit. To further eliminate potential methodological biases, we examined the association between BDNF levels and storage time at $-80^{\circ} \mathrm{C}$ and found no significant association. To our knowledge, no previous studies have explored the effect of sample time after centrifugation. However, a Danish study found plasma BDNF levels to be influenced by centrifugation strategy, which was highly standardised in our analyses. ${ }^{19}$

BDNF levels have not previously been investigated in newly diagnosed patients with bipolar disorder. A possible explanation for our findings could be that the increased BDNF levels that we found are a result of the rapid diagnosis and subsequent medical and psychological treatment in our study. The present patients had a median age at illness onset of 20 years, and a 4 -year median delay in bipolar diagnosis. The majority of the patients were in remission and were not receiving psychotropic medication at the time of inclusion and compared with the healthy control group their consumption of weekly alcohol was significantly lower $(P<0.001)$. These findings could be interpreted as a successful influence of early treatment at a highly specialised affective disorder out-patient clinic, which has a focus on psychiatric medication and group-based psychoeducation including information about the importance of abstaining from alcohol. A previous study from our group showed that patients randomised to the specialised Copenhagen Affective Disorder Clinic were treated with mood stabilisers such as lithium more often, and were substantially less rehospitalised compared with patients randomised to generalised standard treatment. ${ }^{20}$

The above explanation of early diagnosis and intervention resulting in increasing BDNF levels is further supported by a number of other observations. First, BDNF levels increased with illness duration in our study in accordance with similar findings in a prior meta-analysis by our group. ${ }^{9}$ Second, age was positively associated with BDNF levels. This finding is nevertheless in contrast to findings in prior meta-analyses. ${ }^{21,22}$ It should be noted that prior studies included older samples, for example, 11 of the studies included in the meta-regression by Fernandes et $\mathrm{al}^{21}$ had a mean age ranging from 34.0 to 48.6 years as compared with a mean age 31.4 years (median: 29.1 (quartiles: $24.2-36.8$, see Table 1) in the present study.

Third, we found a significant association between higher BDNF levels and anti-epileptic medication. This is in agreement with preclinical trials showing elevated expression of BDNF in hippocampus after lamotrigine injection, ${ }^{23}$ and enhanced BDNF levels with valproate treatment. ${ }^{24}$ This may suggest that early intervention with psychotropic medication in patients with bipolar disorder may prevent early cell death, and thereby enhance neuroplasticity. This possibility is further supported by our finding of no significant difference between patients not receiving any psychotropic medication and healthy controls.

Fourth, we found significantly higher BDNF levels in unaffected first-degree relatives compared with healthy controls. This may function as a compensatory or protective effect of elevated BDNF levels in the premorbid phase in individuals at high risk of bipolar disorder in line with findings in previous studies from our group. ${ }^{25,26}$ Only a few studies have included the unaffected relatives of patients with bipolar disorder. Two studies suggest that unaffected relatives express higher BDNF levels compared with healthy controls without a family history of the disease. ${ }^{27,28}$ Nevertheless, a study examining healthy twins at high and low risk for unipolar and bipolar disorder found no association between BDNF levels and familiar risk, and BDNF levels were not associated with illness onset. ${ }^{29}$

When all participants were split according to gender, the significant difference in BDNF levels between patients with bipolar disorder and healthy controls was only statistically significant within women $(P=0.012)$. This may be because of differences in statistical power (men $n=244$, women $n=425$ ). However, another study from our group ${ }^{26}$ found women to have significantly higher BDNF levels in those individuals with a family risk of affective disorder in accordance with findings in a prior smaller study ${ }^{30}$ but in contrast to others. ${ }^{22}$ We found no significant difference between men and women in any of the three included populations, but, BDNF levels have been found to vary across time in the menstrual cycle. ${ }^{30}$ However, we did not add menstrual cycle as a covariate in the present study.

Notably, most patients in our study were in remission or experienced mild symptoms only reflecting that they were generally well treated. Meta-analyses have consistently found decreased BDNF levels during manic and depressive episodes and normal levels during remission ${ }^{9-11}$ and further that BDNF levels may increase following successful treatment of a manic episode. ${ }^{10}$ In addition, at least two studies that include patients with bipolar disorder who were intensively treated with mood stabilisers have found increased BDNF levels during euthymia. ${ }^{31,32}$ These data may further support the possibility that early medical (and psychological) intervention may add even further to increase BDNF levels as suggested by the present findings.

\section{Strengths and limitations}

The BIO study has a number of substantial advantages. First, the blood sampling and analysis procedures were carried out in a highly standardised way, with all samples taken in a fasting state 
in the morning at a $3.5 \mathrm{~h}$ interval. Second, all samples were stored in the same manner at $-80^{\circ} \mathrm{C}$ until plasma BDNF levels were assayed. Third, all laboratory staff was masked to participant status. Fourth, all patients with newly diagnosed bipolar disorder in the BIO study were first diagnosed clinically by a psychiatrist and then the diagnoses were verified for all participants with a SCAN interview conducted by trained $\mathrm{PhD}$ students in medicine or psychology. Fifth, all patients reported their current use of psychiatric medication, which was validated by trained $\mathrm{PhD}$ students enabling the analysis of the association between current medication status and BDNF levels. Finally, with a median age of illness onset of 20 years and a median delay in bipolar diagnosis of 4 years, the study population is representative of newly diagnosed patients with bipolar disorder. This makes it possible to investigate variables that are unaffected by long-term illness.

There are some limitations to this study. First, the healthy control group could be viewed as a particularly healthy group as they all are recruited through the blood bank, and they had higher education levels compared with patients and the unaffected first-degree relatives. Nevertheless, the blood donors included in this study were recruited from the same catchment area as patients with bipolar disorder, they did not differ in gender composition and differed only slightly in age and educational level from patients, and they were not granted economic compensation for participating. Alternative methods for recruiting control groups include using advertisements or the Danish Civil Registration System. However, both of these methods have relatively low participation response rates and a high risk of selection bias. Taken together, we believe that our control group represents the most reasonable and assessable control group for this study.

Second, although in the fully adjusted model, in addition to subsyndromal symptoms, we included variables such as smoking status and alcohol consumption that may influence BDNF levels, ${ }^{18}$ we cannot exclude effects of unknown or residual confounding.

Third, the number of unaffected first-degree relatives was limited compared with the patient sample, and with a median age of 27 (quartiles: 23-32) years, some may be beyond the risk of onset of bipolar disorder as the risk periods may start during adolescense. Nevertheless, the inclusion of relatives with prior and current depressive episodes increases the heterogeneity of the sample and hence the generalisability of the study. Third, the majority of the patients with bipolar disorder in this study received psychotropic medication during study participation. Consequently, we cannot entirely conclude whether the higher BDNF levels in patients compared with healthy controls is to some extent influenced by medication and not only by the disorder. Only a limited number of patients were not receiving any medication (17.3\%), which may reduce the statistical power of the analyses of non-medicated patients compared with the other groups. Finally, we adjusted for a number of potential relevant confounders.

\section{Implications}

In conclusion, we found increased BDNF levels in newly diagnosed patients with bipolar disorder and their unaffected first-degree relatives compared with healthy controls. Moreover, we found a positive association between BDNF levels, duration of illness, age and use of anti-epileptic medication. Therefore, our findings did not support our hypotheses that patients who have been newly diagnosed with bipolar disorder would have lower BDNF levels compared with healthy controls and that first-degree relatives of patients with bipolar disorder would express intermediary BDNF levels. The results contrast with prior findings of decreased BDNF levels in patients with advanced or progressed bipolar disorder and add to a more complex understanding of the possible influence of neurotrophins on the course of bipolar disorder. Findings from the study may suggest that early diagnosis and intervention may increase BDNF levels and prevent impairment of neuroplasticity.

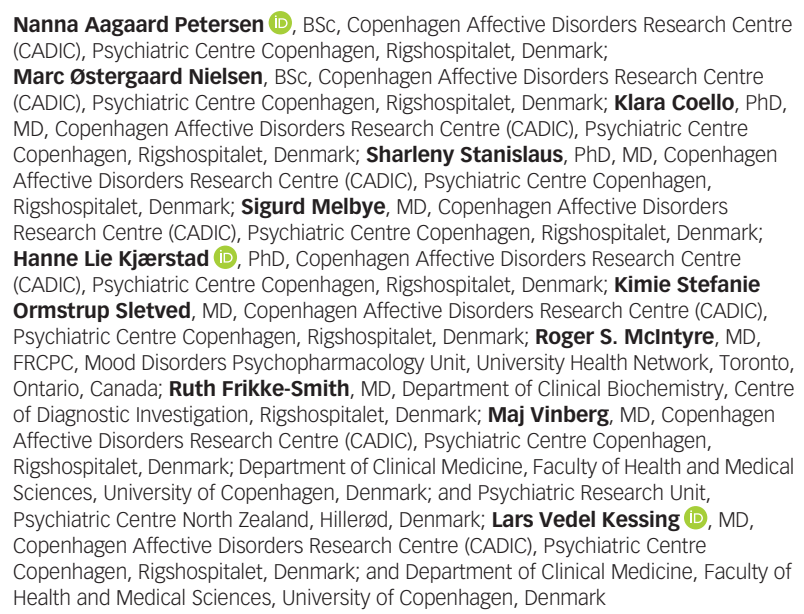

Correspondence: Lars Vedel Kessing. Email: lars.vedel.kessing@regionh.dk

First received 31 Aug 2020, final revision 29 Dec 2020, accepted 14 Jan 2021

\section{Data availability}

Data are available by contacting the corresponding author.

\section{Author contributions}

LV.K. achieved funding and designed the study together with M.V. and N.A.P., K.C., H.L.K., S.S S.M., K.S.O.S. were responsible for participant recruitment, diagnostic and mood assessments, and data collection. N.A.P. conducted the data analysis in cooperation with L.V.K., M.V. and M.Ø.N. N.A.P. wrote the first draft of the manuscript together with M.V. and L.V.K. All authors revised the manuscript and accepted the final version.

\section{Funding}

The study was funded by grants from the Mental Health Services, Capital Region of Denmark, The Danish Council for Independent Research, Medical Sciences (DFF - 4183-00570), Weimans Fund, Markedmodningsfonden (the Market Development Fund, (2015-310), Gangstedfonden (A29594), Helsefonden (16-B-0063), Innovation Fund Denmark (the Innovation Fund, Denmark, 5164-00001B), Copenhagen Center for Health Technology (CACHET), EU H2020 ITN (EU project 722561), Augustinusfonden (16-0083), Lundbeck foundation (R215-2015-4121).

\section{Declaration of interest}

L.V.K. has within the last 3 years been a consultant for Lundbeck and Sunovion. M.V. has within the last 3 years been a consultant for Lundbeck, JanssenCilag and Sunovion. R.S.M. reports research or grants from private industries or non-profit funds: Stanley Medical Research Institute CIHR/GACD/National Natural Science Foundation of China; and consultation/speaker Sunovion, Minerva. R.F.-S. reports consultancy for Novo Nordisk (2019). The remaining authors declare no conflicts of interest.

\section{References}

1 Kessing LV, Andersen PK. Evidence for clinical progression of unipolar and bipolar disorders. Acta Psychiatr Scand 2017; 135: 51-64.

2 Kessing LV, Vradi E, Andersen PK. Life expectancy in bipolar disorder. Bipolar Disord 2015; 17: 543-8.

3 Post RM. Role of BDNF in bipolar and unipolar disorder: clinical and theoretical implications. J Psychiatr Res 2007; 41: 979-90.

4 Manji HK, Duman RS. Impairments of neuroplasticity and cellular resilience in severe mood disorders: implications for the development of novel therapeutics. Psychopharmacol Bull 2001; 35: 5-49.

5 Duman RS, Malberg J, Nakagawa S, D'Sa C. Neuronal plasticity and survival in mood disorders. Biol Psychiatry 2000; 48: 732-9.

6 Pan W, Banks WA, Fasold MB, Bluth J, Kastin AJ. Transport of brain-derived neurotrophic factor across the blood-brain barrier. Neuropharmacology 1998; 37: 1553-61. 
7 Molendijk ML, Spinhoven P, Polak M, Bus BA, Penninx BW, Elzinga BM. Serum BDNF concentrations as peripheral manifestations of depression: evidence from a systematic review and meta-analyses on 179 associations $(\mathrm{N}=9484)$. Mol Psychiatry 2014; 19: 791-800.

8 Fernandes BS, Steiner J, Berk M, Molendijk ML, Gonzalez-Pinto A, Turck CW et al. Peripheral brain-derived neurotrophic factor in schizophrenia and the role of antipsychotics: meta-analysis and implications. Mol Psychiatry 2014; 20: 1108-19.

9 Munkholm K, Vinberg M, Kessing LV. Peripheral blood brain-derived neurotrophic factor in bipolar disorder: a comprehensive systematic review and meta-analysis. Mol Psychiatry 2016; 21: 216-28.

10 Fernandes BS, Molendijk ML, Köhler CA, Soares JC, Leite CMGS, MachadoVieira R, et al. Peripheral brain-derived neurotrophic factor (BDNF) as a biomarker in bipolar disorder: a meta-analysis of 52 studies. BMC Med 2015; 13: 289.

11 Rowland T, Perry BI, Upthegrove R, Barnes N, Chatterjee J, Gallacher D, et al. Neurotrophins, cytokines, oxidative stress mediators and mood state in bipolar disorder: systematic review and meta-analyses. Br J Psychiatry 2018; 213: 514-

12 Kessing LV, Andersen PK. Evidence for clinical progression of unipolar and bipolar disorders. Acta Psychiatr Scand 2017; 135: 51-64.

13 Kessing LV, Munkholm K, Faurholt-Jepsen M, Miskowiak KW, Nielsen LB, FrikkeSchmidt R, et al. The Bipolar Illness Onset study: research protocol for the BIO cohort study. BMJ Open 2017; 7: e015462.

14 American Psychiatric Association. Diagnostic and Statistical Manual of Mental Disorders: DSM-IV (4th edn). APA, 1994.

15 Wing JK, Babor T, Brugha T, Burke J, Cooper JE, Giel R, et al. SCAN: schedules for clinical assessment in neuropsychiatry. Arch Gen Psychiatry 1990; 47: 589-93.

16 Hamilton M. The Hamilton Rating Scale for Depression. In Assessment of Depression (eds N Sartorius, TA Ban): 143-52. Springer Berlin Heidelberg, 1986

17 Young RC, Biggs JT, Ziegler VE, Meyer DA. A rating scale for mania: reliability validity and sensitivity. Br J Psychiatry 1978; 133: 429-35.

18 Bus BA, Tendolkar I, Franke B, de Graaf J, den Heijer M, Buitelaar JK, et al. Serum brain-derived neurotrophic factor: determinants and relationship with depressive symptoms in a community population of middle-aged and elderly people. World J Biol Psychiatry 2012; 13: 39-47.

19 Gejl AK, Enevold C, Bugge A, Andersen MS, Nielsen $\mathrm{CH}_{\text {, Andersen LB. }}$ Associations between serum and plasma brain-derived neurotrophic factor and influence of storage time and centrifugation strategy. Sci Rep 2019; 9 : 9655.

20 Kessing LV, Hansen HV, Hvenegaard A, Christensen EM, Dam H, Gluud C, et al Treatment in a specialised out-patient mood disorder clinic $\mathrm{V}$. standard outpatient treatment in the early course of bipolar disorder: randomised clinical trial. Br J Psychiatry 2013; 202: 212-9.
21 Fernandes BS, Gama CS, Cereser KM, Yatham LN, Fries GR, Colpo G, et al. Brainderived neurotrophic factor as a state-marker of mood episodes in bipolar disorders: a systematic review and meta-regression analysis. J Psychiatr Res 2011; 45: 995-1004.

22 Lommatzsch M, Zingler D, Schuhbaeck K, Schloetcke K, Zingler C, SchuffWerner $\mathrm{P}$, et al. The impact of age, weight and gender on BDNF levels in human platelets and plasma. Neurobiol Aging 2005; 26: 115-23.

23 Li N, He X, Zhang Y, Qi X, Li H, Zhu X, et al. Brain-derived neurotrophic factor signalling mediates antidepressant effects of lamotrigine. Int $J$ Neuropsychopharmacol 2011; 14: 1091-8.

24 Yasuda S, Liang MH, Marinova Z, Yahyavi A, Chuang DM. The mood stabilizers lithium and valproate selectively activate the promoter IV of brain-derived neurotrophic factor in neurons. Mol Psychiatry 2009; 14: 51-9.

25 Knorr U, Sondergaard MHG, Koefoed P, Jorgensen A, Faurholt-Jepsen M, Vinberg $\mathrm{M}$, et al. Increased blood BDNF in healthy individuals with a family history of depression. Psychiatry Res 2017; 256: 176-9.

26 Vinberg M, Trajkovska V, Bennike B, Knorr U, Knudsen GM, Kessing LV. The BDNF Val66Met polymorphism: relation to familiar risk of affective disorder, BDNF levels and salivary cortisol. Psychoneuroendocrinology 2009; 34: 1380-9.

27 Mansur RB, Brietzke E, McIntyre RS, Cao B, Lee Y, Japiassú L, et al. BDNF and $\mathrm{BMI}$ effects on brain structures of bipolar offspring: results from the global mood and brain science initiative. Acta Psychiatr Scand 2017; 136: 607-14.

28 Duffy A, Horrocks J, Doucette S, Keown-Stoneman C, Grof P, Andreazza A, et al. Immunological and neurotrophic markers of risk status and illness development in high-risk youth: understanding the neurobiological underpinnings of bipolar disorder. Int J Bipolar Disord 2014; 2: 29.

29 Vinberg M, Miskowiak K, Kessing LV. Brain Derived Neurotrophic Factor (BDNF) levels as a possible predictor of psychopathology in healthy twins at high and low risk for affective disorder. Psychoneuroendocrinology 2014; 39: 179-83.

30 Piccinni A, Marazziti D, Del Debbio A, Bianchi C, Roncaglia I, Mannari C, et al. Diurnal variation of plasma brain-derived neurotrophic factor (BDNF) in humans: an analysis of sex differences. Chronobiol Int 2008; 25: 819-26.

31 Barbosa IG, Rocha NP, Huguet RB, Ferreira RA, Salgado JV, Carvalho LA, et al Executive dysfunction in euthymic bipolar disorder patients and its association with plasma biomarkers. J Affect Disord 2012; 137: 151-5.

32 Munkholm K, Pedersen BK, Kessing LV, Vinberg M. Elevated levels of plasma brain derived neurotrophic factor in rapid cycling bipolar disorder patients. Psychoneuroendocrinology 2014; 47: 199-211. 